\title{
SPECKLE INTERFEROMETRY
}

\author{
J.C. CHRISTOU \\ Kitt Peak National Observatory \\ National Optical Astronomy Observatories ${ }^{1}$ \\ Box 26732, Tucson AZ 85726-6732, U.S.A.
}

Speckle interferometry is a technique which utilizes the full diffraction-limited imaging potential of ground-based telescopes. Short exposure images, or specklegrams, with an exposure time less than that of the atmospheric correlation time $(\sim 5-50 \mathrm{~ms})$ preserve the high-spatial frequency information lost in long exposure imaging. In 1970, Labeyrie computed the power spectrum of a set of specklegrams and showed that they contained diffraction-limited information. Since then the field has grown with improvements in both instrumentation and the phase recovery algorithms necessary for imaging. It has been applied at both visible and near-infrared wavelengths although, until recently, the latter has used slit-scanning techniques with single pixel detectors because of the lack of array detectors. The current state of speckle interferometry has been well covered in the proceedings of two recent joint National Optical Astronomy Observatories - European Southern Observatory workshops on Interferometric Imaging in Astronomy (Oracle, 1987 \& Garching, 1988).

A number of phase recovery algorithms have been investigated and of these the bispectrum algorithm developed by the Erlangen group is the most attractive as it represents a phase closure technique. However, it is cumbersome in that the bispectrum of a 2-D image is four dimensional. Recent research by a number of groups (Garching, 1988) suggest that it is not necessary to compute the whole bispectrum, especially for visible data, and the advent of faster and larger processors make it attractive. The widely used Knox-Thompson algorithm is a subset of the bispectrum algorithm. Other phase recovery techniques being used include variants on "Shift-and-add" and Speckle Holography. Included in the latter is a technique which simultaneously measures the wavefront and a specklegram. The former can be used to measure an instantaneous point spread function, thus eliminating the requirement of a point source.

Calibration is a major problem in speckle interferometry. Accurate Fourier modulus recovery requires a point source observed under essentially identical seeing conditions. In addition, noise biases contaminate the measured Fourier spectra. For infrared data these biases are detector induced whereas for visible data they come about because of photon statistics. Their removal is not always straightforward with faint object data being affected more severely than bright object data. In addition, detectors contaminate the signal from convolution with a detector response function affected by image tube "pincushion". A

\footnotetext{
${ }^{1}$ Operated by the Association of Universities for Research in Astronomy Inc., under contract with the National Science Foundation
} 
description of these calibration problems is given by Christou (Garching, 1988).

Two types of detectors are currently in use for visible speckle interferometry. These are (i) intensified television systems which record photon events over more than one pixel within a standard $30 \mathrm{~Hz}$ frame, e.g. the Steward Observatory and Erlangen systems, and (ii) the newer generation of individual photon counting systems (IPCS) such as the PAPA and MAMA detectors which also time-tag the events. The latter tend to be more stable and calibratable. At near-infrared wavelengths 2-D detectors are now coming into use, i.e. the NOAO IR speckle camera (Bekers et al., Garching, 1988). The NOAO system uses a $58 \times 62 \mathrm{SBRC}$ InSb array. It is currently limited by readout noise, $\leq 400 \mathrm{e}^{-}$, but as the technology improves larger format detectors with reduced noise will soon be available.

Speckle interferometry has been applied to a broad variety of astronomical objects, from bright objects such as $\alpha$ Orionis to Pluto/Charon $\left(15^{\text {th }}\right.$ magnitude) and the $17^{\text {th }}$ magnitude triple QSO, PG 1115+08. Astrometric measurements of binary and multiple stars represent the majority of the results. In addition there have been measurements of stellar diameters, solar granulation, circumstellar environments and Active Galactic Nuclei. However, until recently, most of these results were obtained through either power spectrum or autocorrelation analysis. With the introduction of improved algorithms, more systematic error free detectors and careful calibration, reliable images have been produced.

A large number of speckle interferometry papers are technical in nature as is evidenced from the NOAO/ESO workshops. However, a perusal through the recent astronomical journals show that there have been $\sim 30$ papers utilizing speckle interferometry results since January 1987. Of these, the majority $(\sim 50 \%)$ report multiplicity. However, a number of these, most notably the one-dimensional infrared results, have not only produced astrometric measurements but also magnitude differences and images. Approximately one half of the other papers report stellar diameters, circumstellar shells etc. Although power spectral analysis is the most common, a number of these papers also show reconstructed images, in particular the MMT and $4 \mathrm{~m}$ measurements of the atmosphere of $\alpha$ Orionis. Most of the other papers have dealt with improved application of the technique. In addition, images of the asteroid 4 Vesta have been reported, the size of SN1987A has been measured at a number of wavelengths and an apparent transient companion has also been detected. The application of speckle interferometry to highly extended objects is also represented with power spectral analysis of solar granulation. The improved understanding of the technique apparent from the workshop proceedings will lead to more imaging papers in the astronomical literature. The use of large aperture instruments such as the family of $4 \mathrm{~m}$ class telescopes and the co-phased MMT $(6.86 \mathrm{~m})$ is already producing images with the same resolution as $2 \mathrm{~cm}$ VLA maps.

Future developments will include (i) the improved resolution of the larger telescopes currently in development, (ii) the larger format detectors especially for near-infrared imaging which will also have lower readout noise and (iii) improved processors which will allow for real-time image reconstructions giving the observer a preliminary idea of the object's morphology. In addition there is improvement of the technique either by the use of wavefront sensing or by the use of partially- or non-redundant apertures, obtained by masking the pupil. Recent results from pupil masking observations suggest improvement in the signal-to-noise ratio.

\section{References}

Proceedings of Joint ESO-NOAO Workshop on Interferometric Imaging in Astronomy, Oracle, January 1987, Ed. J.W. Goad, NOAO, 1987.

Proceedings of Joint NOAO-ESO Conference on High Resolution Imaging by Interferometry, Garching, March 1988, Ed. F. Merkle, ESO, 1988. 